\title{
ALGEBRAS OF OPERATORS AS TOPOLOGICAL ALGEBRAS
}

\author{
F. SADY \\ Institute of Mathematics, University for Teacher Education, 599 Taleghani Avenue, Tehran 15614, I.R. Iran
}

(Received 26 June, 1997)

Introduction. An example of a non-topologizable algebra was given in [2]. In [4] Żelazko gave a simple proof of the fact that, if $X$ is an infinite-dimensional vector space, then the algebra of all finite-rank linear operators on $X$ is not topologizable as a topological algebra. In the following we use a similar idea to prove that, if $E$ is a Fréchet space which is not normable, then each subalgebra $A$ of the algebra of all bounded linear operators on $E$ such that $A$ contains the ideal of continuous, finiterank operators, is non-topologizable as a topological algebra. This is a shorter proof and more general version of the result of [1].

Preliminaries. Let $E$ be a locally convex space, with dual space $E^{\prime}$, and let $\left(p_{\alpha}\right)$ be the family of separating continuous seminorms which defines the topology $\tau$ of $E$. The spaces of all bounded linear operators and all continuous, finite-rank operators on $E$ will be denoted by $\mathcal{B}(E)$ and $\mathcal{F}(E)$, respectively. So $\mathcal{F}(E) \subseteq \mathcal{B}(E)$.

Let $E \otimes E^{\prime}$ be the tensor product of $E$ and $E^{\prime}$, so that $E \otimes E^{\prime}$ is a linear space generated by $\left\{x_{0} \otimes \lambda_{0}: x_{0} \in E, \lambda_{0} \in E^{\prime}\right\}$. Here $x_{0} \otimes \lambda_{0}$ is defined by:

$$
\left(x_{0} \otimes \lambda_{0}\right)(x)=\left\langle x, \lambda_{0}\right\rangle x_{0} \quad(x \in E),
$$

where the notation $\left\langle x, \lambda_{0}\right\rangle$ is used for $\lambda_{0}(x)$. We identify $E \otimes E^{\prime}$ with $\mathcal{F}(E)$.

We recall that a topological algebra $A$ is an associative algebra with a topology on it such that it is a (Hausdorff) topological linear space and the multiplication is jointly continuous.

Now take $A$ to be a subalgebra of $\mathcal{B}(E)$ containing $\mathcal{F}(E)$, and let $A$ be a topological algebra with respect to some topology. Take $\mathcal{V}$ to be a balanced, absorbing, local base for the topology of $A$.

Fix $x_{0} \in E$. Since $E$ is locally convex, there exists $\lambda_{0} \in E^{\prime}$ with $\left\langle x_{0}, \lambda_{0}\right\rangle=1$. Since the topology of $A$ is Hausdorff, there exists $V \in \mathcal{V}$ for which $x_{0} \otimes \lambda_{0} \notin V$. Now choose $W$ in $\mathcal{V}$ with $W^{2} \subseteq V$, and define

$$
K=\operatorname{conv}\left\{x \in E: x \otimes \lambda_{0} \in W\right\} .
$$

Clearly $K$ is convex, absorbing, balanced subset of $E$. So $\rho_{K}$, its Minkowski functional, is a seminorm on $E$. We shall show that $\rho_{K}$ is actually a norm.

For each $\lambda \in E^{\prime}$, there is $m_{\lambda}>0$ such that

$$
x_{0} \otimes \lambda \in m_{\lambda} W .
$$

Now, if $x \in K, x \otimes \lambda_{0} \in W$, and $\lambda \in E^{\prime}$, we have

$$
\left(x_{0} \otimes \lambda\right) \circ\left(x \otimes \lambda_{0}\right) \in m_{\lambda} W^{2} \subseteq m_{\lambda} V .
$$


It is easy to see that

$$
\left(x_{0} \otimes \lambda\right) \circ\left(x \otimes \lambda_{0}\right)=\langle x, \lambda\rangle\left(x_{0} \otimes \lambda_{0}\right)
$$

So $\langle x, \lambda\rangle\left(x_{0} \otimes \lambda_{0}\right) \in m_{\lambda} V$, and, since $x_{0} \otimes \lambda_{0} \notin V$, it follows that $|\langle x, \lambda\rangle| \leq m_{\lambda}$. Therefore $|\langle x, \lambda\rangle| \leq m_{\lambda}$ for each $x \in K$, and consequently

$$
|\langle x, \lambda\rangle| \leq m_{\lambda} \rho_{K}(x) \quad(x \in E) .
$$

This shows that $\rho_{K}$ is a norm because, for each $x \neq 0$ in $E$, there exists $\lambda \in E^{\prime}$ with $\langle x, \lambda\rangle \neq 0$,

Let us write $\|x\|$ for $\rho_{K}(x)$. Rewriting (1) we obtain:

$$
|\langle x, \lambda\rangle| \leq m_{\lambda}\|x\| \quad\left(x \in E, \lambda \in E^{\prime}\right) .
$$

This relation also shows that $B=\{x \in E:\|x\| \leq 1\}$ is a weakly bounded set in $E$. Since $E$ is locally convex, $B$ is bounded. So, for each $\alpha$, there exists $k_{\alpha}>0$ with

$$
p_{\alpha}(x) \leq k_{\alpha}\|x\| \quad(x \in E) .
$$

By replacing $p_{\alpha}$ with $p_{\alpha} / k_{\alpha}$, we can suppose that

$$
p_{\alpha}(x) \leq\|x\| \quad(x \in E)
$$

We can now state our result.

Proposition Let $(E, \tau)$ be a Fréchet space, and let $A$ be a subalgebra of $\mathcal{B}(E)$ containing $\mathcal{F}(E)$. Then there exists a topology on $A$ with respect to which it is a topological algebra if and only if $E$ is a Banach space.

Proof. Let $\|\cdot\|$ and $p_{\alpha}$ be as above, and define

$$
\tilde{p}(x)=\sup _{\alpha} p_{\alpha}(x) \quad(x \in E) .
$$

Then $\tilde{p}(x) \leq\|x\|$, and, since $\left(p_{a}\right)$ is a separating family of seminorms, $\tilde{p}$ is a norm on $E$.

Define $\tilde{B}=\{x \in E: \tilde{p}(x) \leq 1\}$. Then $\tilde{B}$ is an absolutely convex, absorbing set. Since $\tilde{B}=\bigcap_{\alpha}\left\{x \in E: p_{\alpha}(x) \leq 1\right\}$, clearly $\tilde{B}$ is $\tau$-closed. This shows that $\tilde{B}$ is a barrel. Since $(E, \tau)$ is a barrelled space, $\tilde{B}$ contains a neighbourhood of the origin. Hence there exists $\alpha$ such that

$$
\tilde{p}(x) \leq c_{\alpha} p_{\alpha}(x) \quad(x \in E)
$$

for some $c_{\alpha}>0$. Consequently the identity map $i d:(E, \tau) \rightarrow(E, \tilde{p})$, is continuous.

The definition of $\tilde{p}$ shows that $i d:(E, \tilde{p}) \rightarrow(E, \tau)$ is also continuous. Therefore the topology $\tau$ of $E$ as a Fréchet space can be defined by the norm $\tilde{p}$, and so $E$ is a Banach space.

The converse is immediate. 
This proposition shows that for a non-normable, Fréchet space $E$, any subalgebra of $\mathcal{B}(E)$ containing the ideal of continuous, finite-rank operators cannot be topologized as a topological algebra.

For a related result to ours, see [5].

Acknowledgement. This note was prepared during a visit to the University of Leeds in 1997. I am grateful to Professor H.G. Dales for his assistance and hospitality.

\section{REFERENCES}

1. A. Blair, Continuity of multiplication in operator algebras, Proc. Amer. Math. Soc., 6 (1955), 209-210.

2. V. Müller, On topologizable algebras, Studia Math., 99 (1991), 149-153.

3. W. Rudin, Functional analysis, (McGraw-Hill, 1974).

4. W. Żelazko, Concerning topologization of real or complex algebras, Colloq. Math., 71 (1996), 111-113.

5. W. Żelazko, Example of an algebra which is nontopologizable as a locally convex topological algebra, Proc. Amer. Math. Soc., 110 (1996), 947-949. 